\title{
Development and Preliminary Cosmetic Potential Evaluation of Melaleuca alternifolia cheel (Myrtaceae) Oil and Resveratrol for Oily Skin
}

\section{Camila S Oliveira1, Ana Beatriz P P Silva1, Leopoldina L Fagundes', Nadia R B Raposo ${ }^{2}$, Anderson O Ferreira ${ }^{3}$, Marcos Antônio F Brandão ${ }^{2}$ and Hudson C Polonini,1,2,3*}

${ }^{1}$ Faculdade de Ciências Médicas e da Saúde de Juiz de Fora (Suprema), Juiz de Fora, Brazil

${ }^{2}$ Federal University of Juiz de Fora, Juiz de Fora (UFJF), Brazil

${ }^{3}$ Quality Control Laboratory (Ortofarma), Matias Barbosa, Brazil

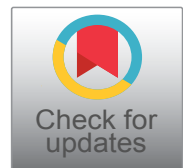

*Corresponding author: Hudson C Polonini, SUPREMA- Faculdade de Ciências Médicas e da Saúde de Juiz de Fora, NUPICS - Núcleo de Pesquisa e Inovação em Ciências da Saúde, Federal University of Juiz de Fora, Juiz de Fora, Ortofarma - Quality Control Laboratories, Matias Barbosa, MG, Brazil, E-mail: hudson@ortofarma.com.br

\begin{abstract}
Background: Oily skin presents shine in excess, as well as increased pores and acne. For this reason, people with oily skin have more difficulty using cosmetics in general. This is the first report in literature to evaluate amulti-purpose dermatological emulsion containing Melaleuca alternifolia Cheel (Myrtaceae) (tea tree) oil and resveratrol for oily skin.

Objectives: This study aimed to develop and evaluate the potential of a cosmetic sunscreen formulation for acne-prone skin containing tea tree oil and resveratrol in volunteers living in Zona da Mata Mineira.

Methods: For photoprotection, in vitro determination tests were performed for Sun Protection Factor (SPF) and UVA Protection Factor (UVAPF). In addition, in vivo tests were carried out for oiliness, hydration, porphyrins, pores and flaking in human volunteers

Results: Most of the volunteers showed improvement in oiliness, porphyrins, hydratation and desquamation by the use of resveratrol and tea tree oil. From the analysis, we obtained the SPF $=15.23$ \pm 576 , UVAPF $=9.0 \pm 1.6$, critical wavelength $=379 \mathrm{~nm}$, and the UVA/UVB ratios were: initial $=0.82$ and final $=0.81$.

Conclusion: The developed product presented moderate UVA and UVB photoprotection (4-stars by Boots Star rating). It also seems to act as an element in the reduction of $P$. acnes by multiple mechanisms, envolving reduction of oiliness, hydration, desquamation and pores sizes.
\end{abstract}

\section{Keywords}

Sunscreen, Resveratrol, Melaleuca oil, Melaleuca alternifolia, Acne, Oily, Acneic skin, Oily skin

\section{Introduction}

Among the types of skin, oily skin needs special care, due to the amount of sebum, mainly on the face. Oily skin may sometimes show signs as excess shine, enlarged pores and acne lesions. For this reason, people with this type of skin have more difficulty using cosmetic products, such as sunscreens [1].

As sunscreens play an important role in the prevention of skin cancer and sun protection, their use in all ages and all types of skin is essential. The development of a sunscreen with high UVA/UVB protection for oily skin is a challenge, since its composition must be specific to this type of skin [2]

We glimpsed an opportunity to carry out this study and aimed to evaluate the effectiveness of an innovative sunscreen for oily skin. In this regard, we selected the raw material of natural origin resveratrol, whose photoprotection had already been described in prior work, besides being an important antioxidant, which has a variable anticancer activity, it also suppresses, slows or reverses the deleterious effects of ultraviolet radiation $[3,4]$.

Another natural raw material also used was Melaleuca alternifolia Cheel (Myrtaceae) oil, popularly known as tea tree, which has antimicrobial, antiseptic and depigmentation actions [5]. Tea tree oil has been used in products for cleansing the skin, especially oily skin, and it is able to eliminate a lot of micro-organisms such as Klebsiella pneumoniae, Lactobacillus spp., Peptostreptococcus anaerobius, Porphyromonas endodentalis, P. gingivalis, Prevotella spp., Propionibacterium acnes, Proteus vulgaris, Pseudomonas aeruginosa, Staphylococcus aureus, among others, besides having great solvent and penetrating power, which helps in the elimination of acne [6]. Within this context, the main objective was to develop and evaluate the potential of cosmetic sunscreen formulations for acneic skin containing Melaleuca alternifolia Cheel (Myrtaceae) oil, resveratrol and zinc oxide, associated or in isolation.

\section{Material and Methods}

\section{Materials}

The materials used for the formulation of the product were: a) melaleuca oil (All Chemistry, lot: 328180); b) edetate (EDTA)

Citation: Oliveira CS, Silva ABPP, Fagundes LL, Raposo NRB, Ferreira AO, et al. (2016) Development and Preliminary Cosmetic Potential Evaluation of Melaleuca alternifolia cheel (Myrtaceae) Oil and Resveratrol for Oily Skin. J Dermatol Res Ther 2:032

Received: July 07, 2016: Accepted: August 29, 2016: Published: August 31, 2016

Copyright: () 2016 Oliveira CS, et al. This is an open-access article distributed under the terms of the Creative Commons Attribution License, which permits unrestricted use, distribution, and reproduction in any medium, provided the original author and source are credited. 
Table 1: Components used in the formulations.

\begin{tabular}{|c|c|c|c|c|}
\hline \multirow[t]{2}{*}{ Component } & \multicolumn{4}{|c|}{ Quantity (\%, m/m) } \\
\hline & F1 & $\mathrm{F} 2$ & F3 & F4 \\
\hline \multicolumn{5}{|l|}{ Phase 1} \\
\hline Sepigel & 5 & 5 & 5 & 5 \\
\hline Chemynol & 0.5 & 0.5 & 0.5 & 0.5 \\
\hline \multicolumn{5}{|l|}{ Phase 2} \\
\hline Water & qs & qs & qs & qs \\
\hline EDTA & 0.1 & 0.1 & 0.1 & 0.1 \\
\hline \multicolumn{5}{|l|}{ Phase 3} \\
\hline Tea tree oil & 2 & & & 2 \\
\hline Resveratrol & & 10 & & 10 \\
\hline Micronized zinc oxide & & & 10 & 10 \\
\hline
\end{tabular}

disodium 99\% (Midelt Chemistry, lot: MD150005); c) phenoxyethanol (and) methylparaben (and) Ethylparaben (and) propylparaben (and) butylparaben (Chemynol, Fagron, lot: 15063517A); d) resveratrol (Fagron, lot: 1510981A); e) polyacrylamide (and) C13-14 Isoparaffin (and) laureth-7 (Sepigel', PharmaNostra, Lot: A15 / 1595AA); f) zinc oxide micronized (PharmaSpecial, lot: Z1P360).

\section{Formulation}

The proposed formulation is a gel-cream, with composition described in table 1.

The compounding processes for the gel-creams were similar to all formulations:

1. The components of Phase 3 were weighed crushed in a mortar and then homogenized.

2. The components of Phase 2 were weighed and mixed to homogeneize.

3. The tea tree oil was added to Phase 3 and mixed to homogenize.

4. Phase 2 was poured into Phase 3.

5. The Sepigel ${ }^{\circledR}$ was weighed and added to a mortar.

6. The mixture of Phases 2 and 3 was poured slowly and gradually over Sepigel $^{\circledR}$, under constant agitation.

7. Chemynol ${ }^{\circledR}$ was added to Step 6, and then the product was homogenized once again.

8. The product was packaged in aluminum tubes.

\section{In vitro determination of the photoprotective potential}

The photoprotection assay followed the protocol described by Colipaand recommended by Brazilian guidelines $[7,8]$. The samples were accurately and quickly weighed (to reduce product evaporation and dryness) to satisfy the application rate of $1.3 \mathrm{mg} \mathrm{cm}^{-2}$ in each PMMA plate (actual quantity applied: $32.5 \mathrm{mg}$, determined by weighing the plates before and immediately after applying the products). They were directly weighed on the plate surface, applied as a large number of small droplets of approximately equal mass, and distributed in an even manner on the roughened surface of the plate. The products were then spread over the whole surface with a fingertip covered with a vinyl glove and pre-saturated with the product to prevent possible losses of the amount weighed. For each product, three plates were kept protected from light exposure in a dark chamber at room temperature $\left(\approx 20^{\circ} \mathrm{C}\right)$ for 15 minutes.

After this period, the plates containing the product were placed in the light-path of the transmittance analyzer. The transmission of UV radiation through the sample was measured from 290 to $450 \mathrm{~nm}$ at $1 \mathrm{~nm}$ intervals at 9 different sites of each plate (total measurement area $\left.=2.0 \mathrm{~cm}^{2}\right)$. The blank was prepared using the HD6 plates covered with $15-\mu \mathrm{L}$ of glycerin, because of its non-fluorescence and UV transparency. For UVAPF, the plates were inserted into the UV irradiation source (temperature maintained below $40^{\circ} \mathrm{C}$ ) and then exposed to a calculated UV dose. After that, new transmission measurements of the sunscreen samples were conducted for acquisition of the second UV spectrum, and then the final UVAPF, the UVA/UVB Ratio and the Critical Wavelength were calculated. Theoretical background can be found in Polonini, et al. [3].

The validation of the results was obtained using the Cosmetics Europe Reference Sunscreen S2 (determined SPF $=18 \pm 1.5$, UVAPF $=12 \pm 1.1, \lambda_{\mathrm{c}}=381 \mathrm{~nm}$, and UVA/UVB Ratio $\left.=0.88\right)$. All results were expressed as a mean of 27 determinations ( 3 plates, 9 readings each, at different sites).

\section{In vivo determination of cosmetic activities}

Volunteers: The present study investigated five volunteers residing in the Zona da Mata of Minas Gerais. Inclusion criteria were: a) healthy subjects; b) healthy skin in the test region; c) agreement to adhere to the procedures and requirements of the study; d) capacity to consent their participation in writing; e) individuals of both sexes and all ethnicities; f) aged between 18 and 40 .

Exclusion criteria were: a) individuals who were using any skin product to control oiliness; b) individuals who have been making use of sunscreen; c) individuals who have declared allergic reaction to components of the formula to be used; d) individuals who had been treated by topical or systemic medication for acne in the 30 days preceding the start of the research.

All study participants used the sunscreen with best results in the in vitro assay for 30 consecutive days $(12 \mathrm{~h} / 12 \mathrm{~h}$ regimen, at $7 \mathrm{am}$ and $19 \mathrm{~h}$ ). The overnight use is justified due to the importance of protection to visible light that are emitted by TVs, mobile phones and laptops.

This study was approved by the Ethics Committee of the Faculdade de CiênciasMédicas da Saúde de Juiz deFora (Suprema) under the number: 1,424.117.

Oiliness, desquamation and pores: In order to determine the oiliness, a Visioscope PC $35^{\circledR}$ (CK Electronic, Germany) that has the function of making visual inspection of any part of the skin was used. To analyze the pores and to quantify oiliness of the skin, we used special Sebufix ${ }^{\circledR}$ adhesives; to quantify the desquamation, Corneofix ${ }^{\circledR}$ adhesives. We visualized regions of the right cheek and forehead, and the quantification was then performed with the aid of the software Complete Skin Investigation (CSI) (CK Electronic, Germany).

Porphyrins: For the determination of porphyrins, we used Visiopor $^{\circledR}$ N PP34 equipment (CK Electronic, Germany). We investigated the forehead, cheeks (left and right) and nose. The device was placed in contact with the skin, the image was captured, and then the analysis was made by the Visiopor software (CK Electronic, Germany).

Hydration: The determination of skin hydration was made using a VapoMeter (Delfin, Finland) after allowing its contact with the skin for 10 seconds. The regions evaluated were the forehead and the right and left cheeks.

\section{Results and Discussion}

The oiliness of the skin is caused by excessive production of sebum by the sebaceous glands, which are usually found in association with a hair follicle - which, together with the gland is referred to as a pilosebaceous unit [1]. The oily skin causes clinical signs that bother and greatly influence the lives of people, and the excess of skin oil can cause from psychological discomforts to skin problems such as acne [8].

Acne vulgaris is a disease of the pilosebaceous follicle that results in clinical inflammatory and non-inflammatory lesions. The pathogenesis of acne is multifactorial, including increased sebum, epidermal hyperproliferation and ductal colonization of the bacterium Propionibacterium acnes, factors that will cause follicular obstruction generating an inflammatory process [9]. P.acnes is normally found in human skin and it is closely associated with acne vulgaris. Lesions are typically mostly inflammatory. Inflammation occurs when P.acnes grows in covered follicles and then releases chemotactic, causing the 
Table 2: Photoprotection activity of F4.

\begin{tabular}{|l|l|l|l|l|}
\hline Parameter & SPF & UVAPF & $\boldsymbol{\lambda C}(\mathbf{n m})$ & UVA / UVB ratio \\
\hline pre irradiation & $12 \pm 2.3$ & $8 \pm 1.4$ & $380 \pm 0.5$ & 0.82 \\
\hline post irradiation & $15 \pm 5.7$ & $8 \pm 2.3$ & $378 \pm 0.3$ & 0.81 \\
\hline
\end{tabular}

SPF $=$ Sun Protection Factor, UVAPF = UVA Protection Factor, $(\lambda \mathrm{c})=$ critical wavelength. Results are expressed as mean \pm standard deviation $(n=27)$.

Table 3: Results of the evaluation of porphyrins in human volunteers.

\begin{tabular}{|c|c|c|c|c|c|c|c|c|}
\hline & \multicolumn{4}{|c|}{ Quantity of porphyrins } & \multicolumn{4}{|c|}{ Size of porphyrins (\%) } \\
\hline & Day 0 & Day 1 & Day 15 & Day 30 & Day 0 & Day 1 & Day 15 & Day 30 \\
\hline \multicolumn{9}{|l|}{ Nose } \\
\hline Volunteer 1 & 56 & 58 & 52 & 36 & 2.02 & 3.50 & 3.22 & 3.77 \\
\hline Volunteer 2 & 66 & 44 & 64 & 59 & 5.95 & 4.36 & 6.80 & 6.50 \\
\hline Volunteer 3 & 68 & 55 & 63 & 69 & 5.41 & 5.03 & 5.56 & 6.72 \\
\hline Volunteer 4 & 101 & 53 & 45 & 58 & 7.70 & 6.29 & 5.01 & 6.39 \\
\hline Volunteer 5 & 58 & 48 & 51 & 53 & 4.11 & 3.26 & 5.50 & 3.28 \\
\hline \multicolumn{9}{|l|}{ Forehead } \\
\hline Volunteer 1 & 24 & 32 & 30 & 36 & 1.32 & 1.28 & 1.82 & 1.67 \\
\hline Volunteer 2 & 4 & 2 & 8 & 4 & 0.07 & 0.07 & 0.23 & 0.09 \\
\hline Volunteer 3 & 35 & 36 & 28 & 39 & 2.14 & 2.07 & 1.39 & 2.06 \\
\hline Volunteer 4 & 63 & 73 & 40 & 47 & 5.82 & 4.67 & 1.96 & 4.58 \\
\hline Volunteer 5 & 4 & 3 & 8 & 4 & 0.09 & 0.04 & 0.28 & 0.16 \\
\hline \multicolumn{9}{|l|}{ Right Cheek } \\
\hline Volunteer 1 & 4 & 4 & 7 & 7 & 0.08 & 0.27 & 0.41 & 0.15 \\
\hline Volunteer 2 & 0 & 2 & 0 & 2 & 0.00 & 0.07 & 0.00 & 0.03 \\
\hline Volunteer 3 & 18 & 43 & 40 & 56 & 1.56 & 2.69 & 3.70 & 3.74 \\
\hline Volunteer 4 & 43 & 40 & 31 & 30 & 5.19 & 4.88 & 3.51 & 2.98 \\
\hline Volunteer 5 & 0 & 1 & 1 & 2 & 0.00 & 0.01 & 0.02 & 0.07 \\
\hline \multicolumn{9}{|l|}{ Left Cheek } \\
\hline Volunteer 1 & 3 & 9 & 9 & 2 & 0.12 & 0.57 & 0.55 & 0.21 \\
\hline Volunteer 2 & 3 & 2 & 0 & 1 & 0.06 & 0.14 & 0.00 & 0.05 \\
\hline Volunteer 3 & 23 & 41 & 39 & 41 & 2.01 & 3.28 & 3.50 & 3.61 \\
\hline Volunteer 4 & 50 & 29 & 30 & 30 & 4.06 & 4.54 & 4.71 & 4.23 \\
\hline Volunteer 5 & 0 & 1 & 1 & 2 & 0.00 & 0.04 & 0.01 & 0.03 \\
\hline
\end{tabular}

Size $(\%)=$ Percentage of the area covered with the fluorescence of porphyrins (red / orange) and other substances (green or yellow color), Quantity: Number of fluorescent spots, porphyrins, in the analyzed area.
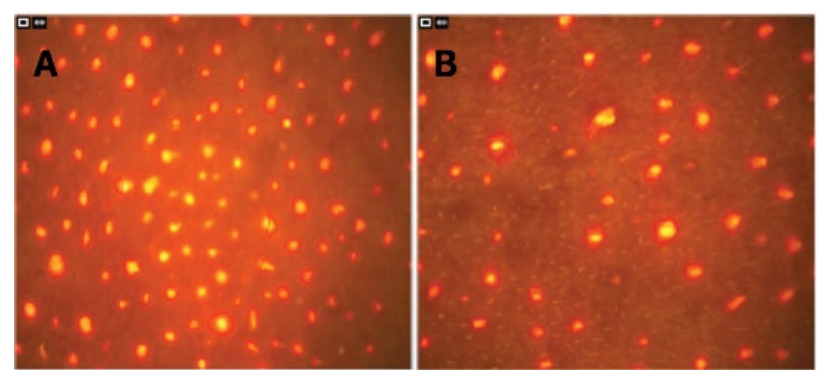

Figure 1: Porphyrins determination.

A) Porphyrins on the nose on day 0; B) Porphyrins on the nose on day 30 (both on the volunteer 4).

inflammatory response, which creates the classic acne comedones [10]. Knowing how this bacterium is involved in the pathogenesis of acne has clinical implications in the treatment of the disease, as it provides a specific direction in order to reach the best results [9].

In the acne treatment, patients should use special products, in order to minimize the problem or solve it [9]. One of the traditional products used is the tea tree oil that is obtained from the leaves of Melaleuca alternifolia, popularly known as tea tree, which can be found in Europe and South America [5]. This oil has antiseptic, antifungal and natural parasiticide properties; in addition, it is extremely effective in combating a wide variety of micro-organisms, including $P$. acnes $[5,11]$. The responsible compounds for this action are their chemical constituents, namely: 1,8-cineole, 1-terpinen-4ol, rho-cymene, linalool, alpha-terpinene, gamma-terpinene, alphaterpineol and terpinolene. The antimicrobial activity is directly related to terpinen-4-ol $[5,11,12]$.
In this study, we used tea tree associated with resveratrol, which is a natural compound produced by some spermatophytes such as grape [10]. It is a powerful antioxidant and anti-inflammatory compound and it has been shown to possess anti-neoplastic activity, as well as capacity to assist in the healing of wounds [13]. It has recently been proven its inhibitory ability to some bacteria, including P.acnes $[10,13]$. In this work, we used the oil as it was received from the manufacturer, so we could mimic the routine of a compounding pharmacy that would prepare the product containing it. This action is of great importance, since it is known that this material frequently suffers adulteration.

Finally, $10 \%$ of micronized zinc oxide was also used, as it has the ability to reflect and disperse the UVB, UVA and visible radiations through an opaque barrier which is formed by particles film on the skin [14]. In a research conducted in MedLine, Scielo and Lilacs, we found no scientific evidence that it influences on skin oiliness.

\section{In vitro assays}

In the preliminary SPF determinations, we found the following: for F1, SPF = 2; for F2, SPF = 7; for F3, SPF = 5; for F4, SPF = 14. As $\mathrm{F} 4$ presented the best result, a complete photoprotective evaluation was performed (Table 2).

From the SPF obtained $(15.23 \pm 5.76)$, it is suggested that the photoprotection obtained arises from the synergistic action of F4 components, because of the results found for the other formulations that contained isolated active constituents. For F3, the result is corroborated by Sunscreen Simulator tool (BASF Pharmaceuticals, www.sunscreensimulator.com), which states that this substance in this concentration possesses an SPF $=4.7$. For its turn, the isolated resveratrol in formulation F2 shows SPF $=7$, which is in accordance to a previous study [3]. It was also expected that the resveratrol did not suffer UV-degradation, as the results in F4 (pre- and post- UV irradiation) did not considerably varied.UVA protection was acceptable, when compared to SPF, as the results were higher than $1 / 3$ of the SPF [15]. The critical wavelength $(\lambda c)$ is another parameter used to evaluate the total capacity of the UVA protection of the product, and it should be greater than $370 \mathrm{~nm}$ [15]. The obtained value was $379 \mathrm{~nm}$, which allows one to affirm that the formulation has broad spectrum protection [7].

The UVA / UVB ratio can also be used to provide the so-called Boots Star Rating, which classifies the level of photoprotection of a product, from 0 to 5 stars. Since it takes into account the results of photoprotection before and after exposure to a radiation source, this classification is important to determine whether the photoprotection values generated are stable, since the components in the formulation can degrade over time and then changes mayoccurin the photoprotective action after irradiation [16]. By Boots Star criteria, the sunscreen received a rating of 4 out of 5 stars.

In the article of Polonini, et al. [3] the results obtained for resveratrol, alone in a formulation, were different from the results found in this study, though the resveratrol was not tested by itself in the formulation. The result in all parameters (critical wavelength, SPF, PFUVA and UVA/UVB ratio) obtained in this formulation were higher than the values found by these authors. When compared to the study of Mercurio [1], which used Anacardiumoccidentale in the formulation and performed the same tests, our result were very different but better, since they were higher. Therefore, it means that the formulation developed in this study offers more protection to the skin.

\section{In vivo assays}

Subclinical presence of P.acnes bacteria is responsible for producing inflammatory mediators, free fatty acids and porphyrins. These porphyrns, when exposed to UV light, emit strong orange-red fluorescence in the follicle openings, thus permitting a visualization and evaluation [17]. The result of the evaluation of porphyrins for 


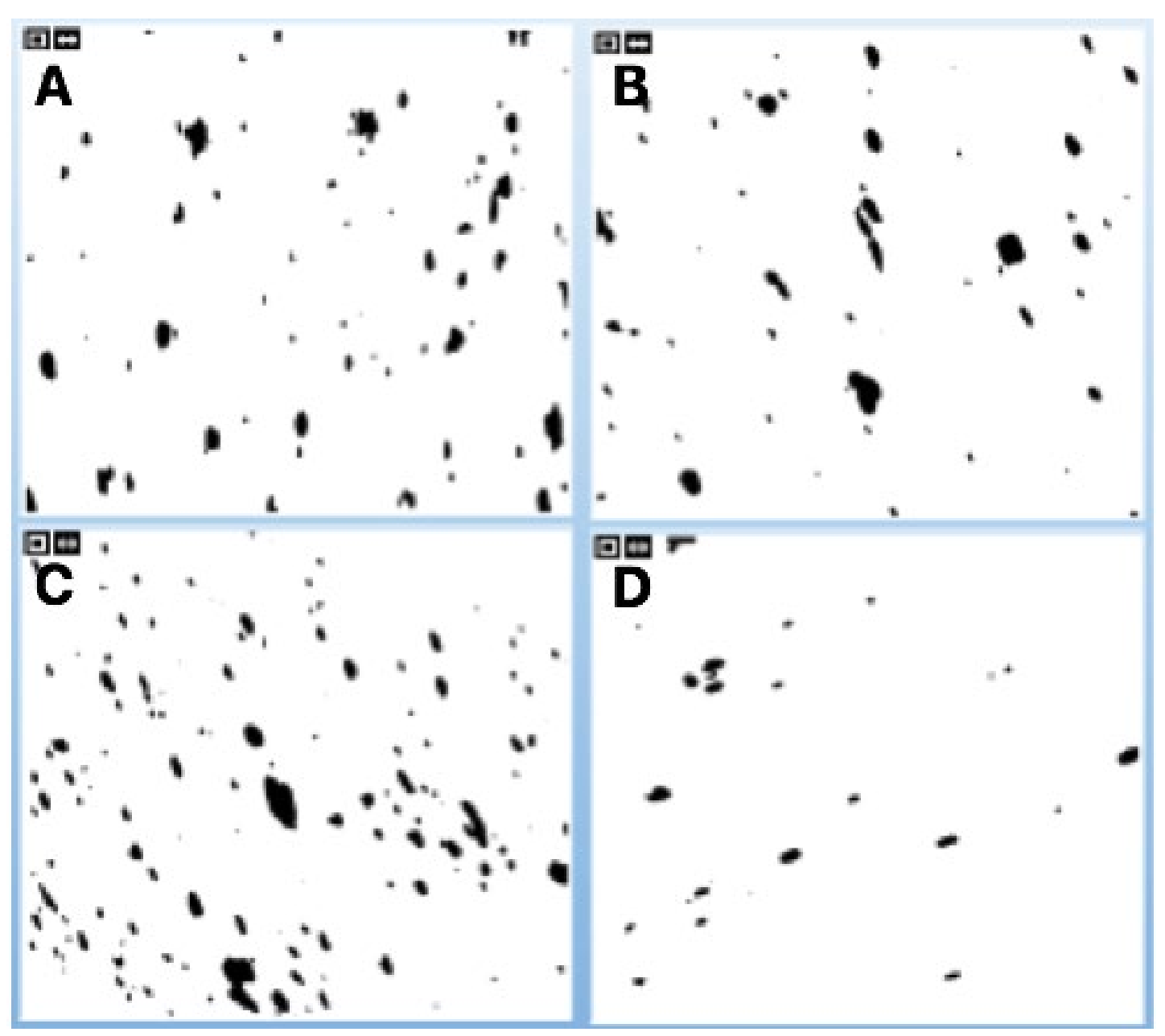

Figure 2: Typical graphic result for determination of oiliness.

A) Test on day 0; B) Test on day 30; C) Right cheek on day 0; D) Right Cheek on 30. All images are from volunteer 3.

Table 4: Results of oiliness evaluation in human volunteers.

\begin{tabular}{|l|l|l|l|l|l|l|l|l|}
\hline \multicolumn{3}{|l}{ Forehead } & \multicolumn{3}{l|}{ Right Cheek } \\
\hline & Day 0 & Day 1 & Day 15 & Day 30 & Day 0 & Day 1 & Day 15 & Day 30 \\
\hline Volunteer 1 & 1.1 & 0.1 & 0.0 & 0.1 & 0.0 & 0.0 & 0.0 & 0.0 \\
\hline Volunteer 2 & 0.1 & 0.1 & 0.1 & 0.1 & 0.0 & 0.2 & 0.0 & 0.0 \\
\hline Volunteer 3 & 2.8 & 2.1 & 1.1 & 2.4 & 4.1 & 1.4 & 2.8 & 0.9 \\
\hline Volunteer 4 & 0.4 & 0.1 & 1.4 & 9.5 & 0.1 & 0.5 & 1.0 & 1.0 \\
\hline Volunteer 5 & 0.1 & 0.4 & 1.7 & 2.3 & 0.0 & 0.0 & 0.0 & 0.0 \\
\hline
\end{tabular}

Table 5: Pore evaluation results in human volunteers.

\begin{tabular}{|l|l|l|l|l|l|l|l|l|}
\hline & \multicolumn{9}{|l}{ Forehead } & \multicolumn{5}{l|}{ Right Cheek } \\
\hline & Day 0 & Day 1 & Day 15 & Day 30 & Day 0 & Day 1 & Day 15 & Day 30 \\
\hline Volunteer 1 & 2.1 & 2.3 & 2.4 & 2.9 & 2.8 & 3.5 & 1.0 & 2.1 \\
\hline Volunteer 2 & 2.3 & 1.8 & 2.2 & 1.7 & 3.6 & 3.3 & 3.6 & 4.8 \\
\hline Volunteer 3 & 2.6 & 2.5 & 1.6 & 3.4 & 4.1 & 2.2 & 3.1 & 2.3 \\
\hline Volunteer 4 & 1.5 & 2.9 & 3.1 & 1.4 & 3.8 & 3.4 & 4.2 & 3.8 \\
\hline Volunteer 5 & 0.9 & 1.2 & 0.5 & 0.9 & 3.4 & 4.8 & 4.5 & 3.7 \\
\hline
\end{tabular}

each volunteer can be seen in table 3. Figure 1 shows the images of the nose area of a porphyrin of volunteers on days 0 and 30 to illustrate typical test results.

The results were conflicting depending on the analyzed area. The nose showed decreased amounts with treatment (e.g., 101 to 58 , or $42.57 \%$ in the volunteer 4 ). For the cheeks, from 50 to 30 , corresponding to $40 \%$ decrease on the left cheek, and from 43 to 30 , a $30.23 \%$ decrease on the right one). The forehead porphyrins decreased up to $25.45 \%$ (in the volunteer 4 ). However, the volunteer 3 had a significant increase (an average of $48.58 \%$ ) in all the analyzed areas.

A smaller number of P.acnes can result in a lower probability of developing acne since it is a causative factor of the disease, but it is not the sole cause. One of the factors for the development of acne is the oiliness, which was the second parameter to be analyzed. The results found during 30 days of continuous use of the sunscreen formulation of each subject are presented in table 4 . In figure 2, a graphical result can be seen.

Volunteer 3 decreased by $14.29 \%$ his oiliness on the forehead, and $78.05 \%$ in the cheek. Volunteers 1, 2 and 5 maintained their oiliness values in at least one of the analyzed areas, suggesting that the product is likely not to increase the production of sebum of the skin even in the presence of oil (tea tree oil) in the formulation.

However, an increase in sebum content in the regions analyzed on volunteers 4 and 5 was observed during the period of 30 days. This effect may have occurred because of a change in temperature conditions on the weeks of the original measures and final measures during the study, since the increased production of sebum may be due to seasonal temperature variations [18].

Quantification of pores was also carried out, since these are directly related to the sebum, which is a very common feature in patients that have oily skin. The results of individual volunteers are shown in table 5. Figure 3 shows the images of the pores of a volunteer on day 0 and on day 30 the area of the right cheek.

After application of the product for 30 days, we could notice a decrease in the values (size) of the pores, it was significantly in at least one of the two face area of all the volunteers (for instance, volunteer 3 showed $43.9 \%$ smaller pores in the left cheek after treatment), which demonstrates the benefit of the application of the formulation during the day.

Regarding the quantification of skin desquamation level, it was 


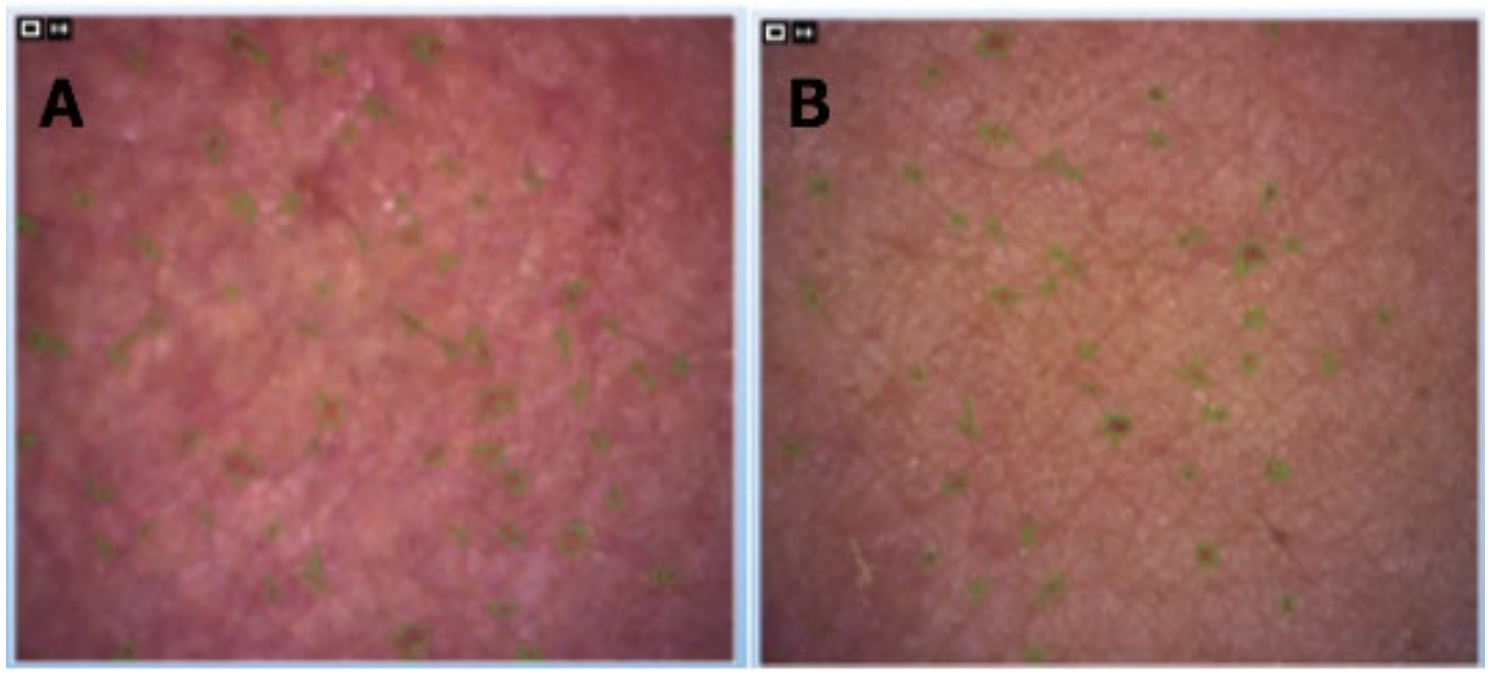

Figure 3: Results typical graph for determining the pores. A) Right Cheek on day 0; B) right cheek 30 on both the voluntary 3 . The pores can be displayed by the green marking.
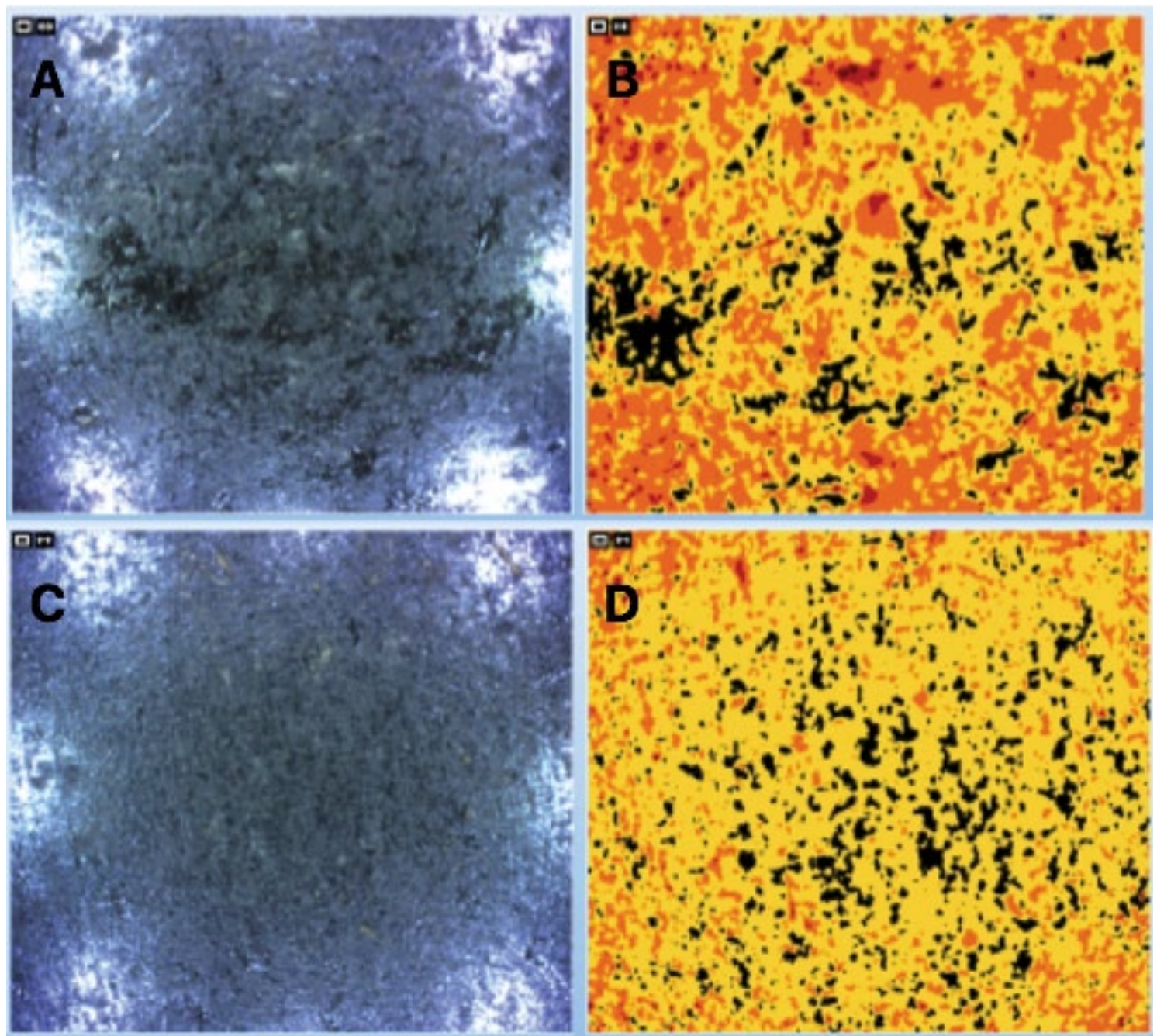

Figure 4: Typical graphic result for determination of desquamation. A,B) Volunteer 3, forehead, day 0; C,D) Volunteer 3 , forehead, day 30.

Table 6: Results of evaluation of desquamation on human volunteers.

\begin{tabular}{|l|l|l|l|l|l|l|l|l|}
\hline & \multicolumn{9}{|l}{ Forehead } \\
\hline & Day 0 & Day 1 & Day 15 & Day 30 & Day 0 & Day 1 & Day 15 & Day 30 \\
\hline Volunteer 1 & 32.87 & 35.33 & 35.37 & 36.09 & 30.88 & 28.65 & 34.93 & 29.25 \\
\hline Volunteer 2 & 31.68 & 35.53 & 29.99 & 33.45 & 32.42 & 33.10 & 31.76 & 36.08 \\
\hline Volunteer 3 & 36.69 & 36.17 & 29.45 & 31.84 & 35.16 & 36.19 & 31.24 & 34.87 \\
\hline Volunteer 4 & 31.70 & 32.26 & 31.77 & 31.55 & 35.38 & 34.20 & 34.69 & 32.06 \\
\hline Volunteer 5 & 35.28 & 32.56 & 37.90 & 31.42 & 37.02 & 33.37 & 36.72 & 33.61 \\
\hline
\end{tabular}

observed a decrease in this feature in the volunteers, as it is shown in table 6 and figure 4 .

These values show that most of the patients showed a reduction in skin desquamation. In volunteer 3 , the difference was $13.22 \%$ and $0.82 \%$ on the forehead on the right cheek, respectively; in volunteer 4, this difference was $0.47 \%$ and $9.38 \%$; and in volunteer $5,10.94 \%$ and $9.21 \%$. For his turn, the volunteer 2 showed increments of $5.59 \%$ and $11.29 \%$ in the same areas. In an overview, this result proves to 
Table 7: Results of the evaluation of hydration in human volunteers.

\begin{tabular}{|c|c|c|c|c|}
\hline \multirow[b]{3}{*}{ Forehead } & \multicolumn{4}{|c|}{ Transepidermal water loss $\left(\mathrm{mg} \mathrm{cm}^{-2} \mathrm{~h}^{-1}\right)$} \\
\hline & \multicolumn{4}{|c|}{ Forehead } \\
\hline & Day 0 & Day 1 & Day 15 & Day 30 \\
\hline Volunteer 1 & 17.0 & 18.4 & 17.1 & 43.4 \\
\hline Volunteer 2 & 15.6 & 24.3 & 29.0 & 20.2 \\
\hline Volunteer 3 & 21.8 & 17.2 & 19.1 & 14.4 \\
\hline Volunteer 4 & 23.9 & 31.6 & 31.3 & 29.3 \\
\hline Volunteer 5 & 27.9 & 37.1 & 17.1 & 18.9 \\
\hline \multicolumn{5}{|l|}{ Right Cheek } \\
\hline Volunteer 1 & 13.8 & 15.5 & 15.6 & 31.8 \\
\hline Volunteer 2 & 12.5 & 18.0 & 25.4 & 30.7 \\
\hline Volunteer 3 & 18.7 & 22.2 & 19.6 & 21.4 \\
\hline Volunteer 4 & 21.0 & 23.8 & 22.0 & 51.2 \\
\hline Volunteer 5 & 16.4 & 16.2 & 12.5 & 14.5 \\
\hline \multicolumn{5}{|l|}{ Left Cheek } \\
\hline Volunteer 1 & 12.7 & 14.0 & 15.7 & 31.6 \\
\hline Volunteer 2 & 11.8 & 21.1 & 33.9 & 25.4 \\
\hline Volunteer 3 & 21.7 & 23.1 & 19.7 & 20.4 \\
\hline Volunteer 4 & 19.4 & 24.3 & 23.4 & 22.2 \\
\hline Volunteer 5 & 15.8 & 13.4 & 12.2 & 14.5 \\
\hline
\end{tabular}

be positive, when compared to similar studies that performed the same tests we did [1]: it was seen that the desquamation of the skin is proportional to the amount of bacteria $P$. acnes- causing the bacteria number to decrease.

Finally, skin hydration evaluation was performed. Hydration is the direct result of the maintenance of the water within the epidermis. The results of each volunteer is shown in table 7.

In terms of transepidermal water loss, it can be seen that there were large variations between the results of the volunteers; most of which had an increase in the water loss, with the exception of volunteer 5 who showed a decrease in the value $(32.26 \%$ on the forehead, $11.59 \%$ in the right cheek and $8.23 \%$ in the left cheek) Except for volunteer 5, the results indicate that there was a worsening of hydration in the other volunteers. This suggests that the product may have a negative effect on the hydration of the skin, which can be linked to the fact that oily skin naturally has a lower hydration. As the formulation F4 seems to act decreasing sebum production in skin, it will consequently increase the transepidermal loss of water.

In the study of Mercurio [1], a formulation containing Anacardium occidentale was developed and it was analyzed all the parameters by the same methods used in this article. The results obtained were very similar, but the formulation containing tea tree was more efficient when controlling oiliness of the skin, and that is the parameter of greatest interest in the study.

\section{Conclusion}

The main findings of this work were: (i) formulation F4 presented moderate UVA and UVB photoprotection (4-stars by Boots Star rating), and this is the first report to show the photoprotective potential of tea tree and resveratrol in association; (ii) F4 reduces the amount of porphyrins in the face of the human volunteers, confirming its antibacterial action against $P$. acnes; and (iii) the product seems to act in the control of acne by multiple mechanisms, envolving reduction of oiliness, hydration, desquamation and pores sizes. Given this, F4 seems to be a good candidate to be a multi-purpose dermatological product, comprising photoprotection, reduction in acne vulgaris and oiliness.

\section{References}

1. Mercurio DG (2012) Desenvolvimento e avaliação de eficácia de formulações fotoprotetoras para a pele oleosa contendo extrato de Anacardiumoccidentale. Universidade de São Paulo, Tese de Doutorado.

2. Campos PMBGM, Mercurio DG (2009) Farmacologia e a pele. Rev Bras Med 66:15-21.

3. Polonini HC, Lima LL, Gonçalves KM, Carmo ARM, Silva AD, et al. (2013) Photoprotective activity of resveratrol analogues. Bioorg Med Chem 21: 964968.

4. Chan CM, Huang CH, Li HJ, Hsiao CY, Su CC, et al. (2015) Protective effects of resveratrol against UVA-induced damage in ARPE19 cells. Int J Mol Sci 16: $5789-5802$.

5. Baccoli BC, Sciani MD, Carvalho (2015) Os benefícios do óleo de melaleuca na acne grau II e III: uma revisão de literatura. Revista da Universidade Vale do Rio Verde13: 536-547.

6. (2012) Regulamento Técnico Mercosul sobre Protetores Solares em Cosméticos. RDC No 30 .

7. (2011) The European Cosmetic, Toiletry and Perfumery Association (COLIPA). Method for in vitro determination of UVA protection.

8. Youn SW, Park ES, Lee DH, Huh CH, Park KC (2005) Does facial sebum excretion really affect the development of acne? Br J Dermatol 153: 919-924.

9. Dessinioti C, Katsambas AD (2010) The role of Propionibacterium acnes in acne pathogenesis: facts and controversies. Clin Dermatol 28: 2-7.

10. Docherty JJ, McEwen HA, Sweet TJ, Bailey E, Booth DT (2007) Resveratrol inhibition of Propionibacterium acnes. Journal of antimicrobial chemotherapy 59: $1182-1184$

11. Carson Cf, Hammer KA, Riley TV (2006) Melalelucaalternifolia (tea tree) oil: a review of antimicrobial and other medicinal properties. Clin Microbiol Rev 19: 60-62.

12. Cox SD, Mann Cm, Markham JL, Bell HC, Gustafson JE, et al. (2000) The mode of antimicrobial action of the essencial oil of Melaleuca alternifolia (tea tree). J Appl Microbiol 88: 170-175.

13. Taylor EJ, Yu Y, Champer J, Kim J (2014) Resveratrol demonstrates antimicrobial effects against Propionibacterium acnes in vitro. Dermatol Ther 4: 249-257.

14. Balogh TS, Velasco MVR, Pedriali CA, Kaneko TM, Baby AR (2011) Ultraviolet radiation protection: current available resources in photoprotection. An Bras Dermatol 86: 732-742.

15. (2010) Regulamento Técnico MERCOSUL Sobre Protetores Solares em Cosméticos. Portaria No 2.466 .

16. Boots the Chemist Ltd (2008) The revised guidelines to the practical measurement of UVA/UVB ratios according to the Boots Star Rating System. The Boots Co, Nottingham, PLC.

17. (2014) Information and operating instruction for the Visiopor ${ }^{\circledR}$ PP34 with software. Köln.

18. Youn SW, Na JI, Choi SY, Huh HC, Park KC (2005) Regional and seasonal variations in facial sebum secretions: a proposal for the definition of combination skin type. Skin Res Technol 11: 189-195. 